\title{
Pulse Fluoroscopy Radiation Reduction in a Pediatric Cardiac Catheterization Laboratory
}

\author{
Stuart H. Covi, MD, ${ }^{*}$ Wendy Whiteside, MD, ${ }^{\dagger}$ Sunkyung Yu, MS, ${ }^{\dagger}$ and Jeffrey D. Zampi, $\mathrm{MD}^{\dagger}$ \\ *Department of Pediatrics, and 'Division of Pediatric Cardiology, Department of Pediatrics, University of Michigan, Ann \\ Arbor, Mich, USA
}

\section{A B S T R A C T}

Objective. To determine if lower starting pulse fluoroscopy rates lead to lower overall radiation exposure without increasing complication rates or perceived procedure length or difficulty.

Setting. The pediatric cardiac catheterization laboratory at University of Michigan Mott Children's Hospital.

Patients. Pediatric patients with congenital heart disease.

Design/Interventions. We performed a single-center quality improvement study where the baseline pulse fluoroscopy rate was varied between cases during pediatric cardiac catheterization procedures.

Outcome Measures. Indirect and direct radiation exposure data were collected, and the perceived impact of the fluoroscopy rate and procedural complications was recorded. These outcomes were then compared among the different set pulse fluoroscopy rates.

Results. Comparing pulse fluoroscopy rates of $15,7.5$, and 5 frames per second from 61 cases, there was a significant reduction in radiation exposure between 15 and 7.5 frames per second. There was no difference in perceived case difficulty, procedural length, or procedural complications regardless of starting pulse fluoroscopy rate.

Conclusions. For pediatric cardiac catheterizations, a starting pulse fluoroscopy rate of 7.5 frames per second exposes physicians and their patients to significantly less radiation with no impact on procedural difficulty or outcomes. This quality improvement study has resulted in a significant practice change in our pediatric cardiac catheterization laboratory, and 7.5 frames per second is now the default fluoroscopy rate.

Key Words. Congenital Heart Disease; Pediatric Diagnostic Catheterization; Patient-Centered Outcomes Research; Radiation Physics; Dosimetry

\section{Introduction}

$\mathrm{C}$ ardiac catheterizations constitute a major source of radiation exposure for patients with congenital heart disease, particularly those patients requiring repeated procedures for either diagnostic, interventional, or biopsy-related reasons. As children are up to 10 times more sensitive to the stochastic effects of ionizing radiation and have a longer life expectancy compared to adults, there is potential for repeated exposure and manifestation of the ill effects of radiation exposure. ${ }^{1}$ Thus, efforts to reduce radiation dose are an important aspect of improving patient safety during pediatric cardiac catheterization procedures.

One potential way to lower radiation exposure is to lower pulse fluoroscopy rate (PFR). Histori-

(C) 2014 Wiley Periodicals, Inc. cally, changing from conventional "continuous" (interlaced) fluoroscopy to pulsed fluoroscopy resulted in a $42 \%$ exposure reduction and thereby revealed the importance of balancing quality of imaging with amount of radiation exposure. ${ }^{2}$ In the adult population, lower PFR has been shown to further reduce radiation dose to patients. ${ }^{3}$ However, because of their higher heart rates, lowering PFR in children could result in longer fluoroscopy times due to poor visualization of cardiac structures.

The purpose of this study was to evaluate the effect of lower PFR on radiation exposure and image quality in a pediatric cardiac catheterization laboratory in an effort to initiate change in physician radiation practice patterns. We hypothesized that lower starting PFR would result in lower

Congenit Heart Dis. 2015;10:E43-E47 
radiation exposure without compromising the ability to complete the cardiac catheterization, lengthening the procedure, or causing procedural complications.

\section{Materials and Methods}

Between March 2013 and May 2013, all diagnostic and interventional catheterizations performed in the pediatric cardiac catheterization laboratory at the University of Michigan Congenital Heart Center by three primary interventional cardiology attendings were included in the study. Cases involving only placement of central venous catheters were excluded. Prior to each case, the baseline PFR was selected randomly within each physician's preexisting "standard" and "low-dose" practice patterns. Because of variation in each individual physician's typical radiation practice patterns, the "standard" PFR was either 15 or 7.5 frames per second (fps), and the "low" PFR was either 7.5 or $5 \mathrm{fps}$, thus creating 3 patient groups: $15 \mathrm{fps}, 7.5 \mathrm{fps}$, and 5 fps. Physicians were encouraged to change the PFR during the procedure as they felt necessary for appropriate patient care.

To monitor radiation exposure, patient dose was indirectly recorded using standard techniques, including total fluoroscopy time (minutes), air kerma (mGy), and dose area product ( $\mu \mathrm{Gy} \cdot \mathrm{m} 2)$. Additionally, physician radiation exposure was directly measured (mrem) using Instadose dosimetry badges (Mirion Technologies, Inc., Smyrna, GA, USA), which were placed on the outside collar of the standard lead apron for every physician in the procedure (attending interventionalists and pediatric cardiology fellows). After each case, these badges were analyzed for the highest individual dosimeter dose, and the average of the two highest dosimeter dosages was recorded without physician knowledge of the result. Due to similarities between these numbers, only the averages of the two highest dosimeter dosages are presented in this paper.

At the completion of the case, the attending physician was asked to fill out a survey to assess various case factors, including degree of case difficulty as well as the impact of the set PFR on the case difficulty and outcome of the procedure. Case complexity was assessed using an aggregate complexity score (maximum difficulty $=12$ ), which was composed of individual complexity scores for vascular access (maximum difficulty $=4$ ), the diagnostic portion of the procedure (maximum difficulty $=$
4), and the interventional portion of the procedure (maximum difficulty $=4$ ). The maximum possible case complexity score for diagnostic cases was therefore 8, and for interventional cases it was 12 . Additionally, the number of angiograms performed, changes in fluoroscopy rate carried out by the user, and the use of lead shielding were recorded by the study team at the conclusion of each case.

Group comparisons between starting PFR groups were made in patient and procedural characteristics using chi-square test or Fisher's exact test for categorical variables and Wilcoxon ranksum test for continuous variables. Similar comparisons were also made between providers in the 7.5 -fps group to examine the impact of practice variability. The independent association of starting PFR with radiation exposure was determined using multivariable logistic regression controlling for case type and number of angiograms. In the logistic regression, radiation exposure was evaluated as a binary outcome using the 25 th percentile or median value. The adjusted odds ratio and $95 \%$ confidence interval obtained from logistic regression were reported. Lastly, a correlation between direct and indirect methods of measuring radiation exposure was evaluated using the Spearman correlation coefficient. Analysis was based on the intent-to-treat principle. All statistical analysis was performed using SAS version 9.3 (SAS Institute Inc, Cary, NC, USA). $P$ values less than .05 were considered statistically significant.

This study was approved with exempt status by our institutional review board.

\section{Results}

Over the study period, 61 procedures were included in the analysis (starting PFRs of $15 \mathrm{fps}$ $[\mathrm{n}=20], 7.5 \mathrm{fps}[\mathrm{n}=25]$, and $5 \mathrm{fps}[\mathrm{n}=16])$. There were no differences in baseline demographics and case complexity between PFR groups. There was, however, a significant difference in case type between groups, with more diagnostic cases in the 15 -fps group and more interventional cases in the 7.5-fps group (Table 1).

A starting PFR of 7.5 fps was associated with a significant reduction in radiation exposure compared to 15 fps (Table 1), which remained significant after controlling for case type and number of angiograms performed (Table 2). There was no difference in radiation exposure between 7.5 and 5 fps. 
Table 1. Comparison of Patient and Clinical Characteristics between Initial Pulse Fluoroscopy Rate Groups ( $\mathrm{n}=61$ )

\begin{tabular}{|c|c|c|c|c|c|}
\hline \multirow[b]{2}{*}{ Characteristics } & \multicolumn{3}{|c|}{ Initial fluoroscopy rate (fps) } & \multicolumn{2}{|l|}{$P$ value } \\
\hline & $5(n=16)$ & $7.5(n=25)$ & $15(n=20)$ & 5 vs. 7.5 & 7.5 vs. 15 \\
\hline Age at procedure (years), median (IQR) & $4.5(1.8-13.7)$ & $2.7(0.5-7.7)$ & $3.8(1.3-16.6)$ & .19 & .10 \\
\hline Weight $(\mathrm{kg})$, median (IQR) & $13.9(10.9-45.2)$ & $12.1(6.3-23.2)$ & $15.6(10.3-55.0)$ & .16 & .14 \\
\hline Body surface area $\left(\mathrm{m}^{2}\right)$, median (IQR) & $0.61(0.49-1.4)$ & $0.53(0.31-0.95)$ & $0.67(0.46-1.54)$ & .23 & .17 \\
\hline \multicolumn{6}{|l|}{ Case type, $n(\%)^{\star}$} \\
\hline Diagnostic & $8(50.0)$ & $9(36.0)$ & $14(70.0)$ & .33 & .01 \\
\hline Intervention & $7(43.8)$ & $15(60.0)$ & $4(20.0)$ & & \\
\hline Biopsy & $1(6.3)$ & $1(4.0)$ & $2(10.0)$ & & \\
\hline \multicolumn{6}{|l|}{ Case complexity score, median (IQR) } \\
\hline Diagnostic procedures & $4(2.5-4.5)$ & $3.5(2.5-4.5)$ & $4(3-5)$ & .79 & .26 \\
\hline Interventional procedures & $6(5-7)$ & $5(5-7)$ & $5.5(3.5-7.5)$ & .64 & .80 \\
\hline $\begin{array}{l}\text { Radiation exposure (mrem), average of } \\
\text { two highest dosimeter readings (IQR) }\end{array}$ & $2.3(0-5.8)$ & $2(0-4)$ & $4.3(2.3-11.5)$ & .54 & .0496 \\
\hline
\end{tabular}

${ }^{*}$ Comparison made only between diagnostic and interventional case types, given low number of biopsy cases.

$\mathrm{IQR}$, interquartile range.

Table 2. Association between Radiation Exposure and Initial Pulse Fluoroscopy Rate Controlling for Number of Angiograms and Case Type $(n=61)$

\begin{tabular}{|c|c|c|c|c|}
\hline \multirow[b]{3}{*}{ Initial fluoroscopy rate (fps) } & \multicolumn{4}{|c|}{ Radiation exposure (mrem) $^{*}$} \\
\hline & \multicolumn{2}{|c|}{$>0$ (25th percentile) } & \multicolumn{2}{|l|}{$\geq 3$ (median) } \\
\hline & AOR $(95 \% \mathrm{Cl})$ & $P$ & $\operatorname{AOR}(95 \% \mathrm{Cl})$ & $P$ \\
\hline 7.5 vs. 5 & $0.59(0.12-3.03)$ & .53 & $1.07(0.22-5.17)$ & .93 \\
\hline 15 vs. 7.5 & $7.52(1.15-49.0)$ & .03 & $7.00(1.29-37.9)$ & .02 \\
\hline
\end{tabular}

Patients undergoing biopsy were not included in the model due to small sample size.

${ }^{*}$ Average of two highest physician dosimeters.

AOR, adjusted odds ratio; $\mathrm{Cl}$, confidence interval.

Table 3. Perceived Impact of Initial Pulse Fluoroscopy Rate on Procedural Difficulty and Procedure Length $(n=61)$

\begin{tabular}{|c|c|c|c|c|c|}
\hline \multirow[b]{2}{*}{ Responses to questions, n (\%) } & \multicolumn{3}{|c|}{ Initial fluoroscopy rate (fps) } & \multicolumn{2}{|l|}{$P$ value ${ }^{\star}$} \\
\hline & $5(n=16)$ & $7.5(n=25)$ & $15(n=20)$ & 5 vs. 7.5 & 7.5 vs. 15 \\
\hline \multicolumn{6}{|c|}{$\begin{array}{l}\text { "Did the starting fluoroscopy rate impede your ability to complete the } \\
\text { procedure as planned?" }\end{array}$} \\
\hline Definitely not & $15(93.8)$ & $23(92.0)$ & $20(100.0)$ & 1.00 & 1.00 \\
\hline Probably not & $0(0.0)$ & $1(4.0)$ & $0(0.0)$ & & \\
\hline Possibly & $0(0.0)$ & $0(0.0)$ & $0(0.0)$ & & \\
\hline Definitely & $1(6.3)$ & $1(4.0)$ & $0(0.0)$ & & \\
\hline \multicolumn{6}{|c|}{ "Did the starting fluoroscopy rate increase the length of the procedure?" } \\
\hline Definitely not & $15(93.8)$ & $22(88.0)$ & $17(85.0)$ & 1.00 & 1.00 \\
\hline Probably not & $0(0.0)$ & $1(4.0)$ & $1(5.0)$ & & \\
\hline Possibly & $0(0.0)$ & $1(4.0)$ & $0(0.0)$ & & \\
\hline Definitely & $1(6.3)$ & $0(0.0)$ & $0(0.0)$ & & \\
\hline Unknown & $0(0.0)$ & $1(4.0)$ & $2(10.0)$ & & \\
\hline
\end{tabular}

${ }^{*}$ Comparisons of difficulty/length were made as definitely not/probably not vs. possibly/definitely.

There were no significant differences in physician-perceived ability to complete the procedure or impact of PFR on the procedural length between any of the PFR groups (Table 3). There were no complications observed during the study and therefore no complications attributable to PFR.

In order to determine interphysician variability within the 7.5 -fps group, we compared providers and found both patient characteristics and case type/complexity to be similar. Despite the use of more radiation-blocking techniques for one physician, there were no significant differences in radiation exposure between providers (Table 4).

Comparing the indirect and direct methods of measuring radiation exposure, a positive correlation was found between all the indirect measures of patient radiation exposure and the direct measurement of physician radiation exposure using the case-specific dosimetry badges (Table 5). 
Table 4. Comparison of Patient and Clinical Characteristics between Providers at the Initial Fluoroscopy Rate of $7.5 \mathrm{fps}$ $(\mathrm{n}=25)$

\begin{tabular}{|c|c|c|c|}
\hline Characteristics & Provider $1(n=14)$ & Provider $2(n=11)$ & $P$ value \\
\hline Age at procedure (years), median (IQR) & $2.5(0.8-11.3)$ & $2.7(0.01-7.7)$ & .63 \\
\hline Weight $(\mathrm{kg})$, median (IQR) & $11.9(7.0-36.8)$ & $12.2(3.7-23.2)$ & .75 \\
\hline Body surface area $\left(\mathrm{m}^{2}\right)$, median (IQR) & $0.5(0.4-1.2)$ & $0.5(0.2-1.0)$ & .75 \\
\hline \multicolumn{4}{|l|}{ Case type, $n(\%)^{*}$} \\
\hline Diagnostic & $6(42.9)$ & $3(27.3)$ & .42 \\
\hline Intervention & $7(50.0)$ & $8(72.7)$ & \\
\hline Biopsy & $1(7.1)$ & $0(0.0)$ & \\
\hline \multicolumn{4}{|l|}{ Case complexity score, median (IQR) } \\
\hline Diagnostic procedure & $3(3-4)$ & $4(2-6)$ & .66 \\
\hline Interventional procedure & $6(5-7)$ & $5(3.5-7.5)$ & .42 \\
\hline Usage of additional lead shielding, $n(\%)$ & $7(50.0)$ & $0(0.0)$ & .01 \\
\hline Radiation exposure (mrem), average of two highest dosimeter readings (IQR) & $2.8(0-6)$ & $0(0-3)$ & .15 \\
\hline
\end{tabular}

Because of the similar practice patterns of two providers, their data were grouped together and presented as one dataset.

${ }^{*}$ Comparison was made between diagnostic and interventional case types given low number of biopsy cases group.

Table 5. Correlation between Direct and Indirect Measures of Radiation Exposure $(n=61)$

\begin{tabular}{|c|c|c|}
\hline \multirow{2}{*}{$\begin{array}{l}\text { Indirect measurements of total } \\
\text { radiation exposure }\end{array}$} & \multicolumn{2}{|c|}{$\begin{array}{l}\text { Direct measurement of radiation } \\
\text { exposure }(\mathrm{mrem})^{\star}\end{array}$} \\
\hline & $r$ & $P$ value \\
\hline Minutes & 0.43 & .0005 \\
\hline mGy & 0.64 & $<.001$ \\
\hline mGy/BSA & 0.55 & $<.001$ \\
\hline $\mathrm{mGy} / \mathrm{min}$ & 0.49 & $<.001$ \\
\hline $\mathrm{mGy} / \mathrm{min} / \mathrm{BSA}$ & 0.32 & .01 \\
\hline Dose area product & 0.60 & .0003 \\
\hline
\end{tabular}

${ }^{*}$ Radiation dose to physicians measured using dosimetry badges.

†Fluoroscopy equipment-derived measurement of patient radiation exposure. ‡Only measured for subset of population (32/61, 52\%).

$\mathrm{BSA}$, body surface area; $r$, Spearman correlation coefficient.

\section{Discussion}

Similarly to what has been reported in the adult cardiac catheterization literature, this quality improvement study showed that lowering the PFR from 15 to 7.5 fps was associated with a significant decrease in measured direct radiation exposure. While our study focused on PFR, a significant amount of radiation exposure during cardiac catheterizations occurs during cineangiography due to higher frame rates and higher energy and radiation doses, as well as due to longer procedural duration during interventional cases. Yet we found that the radiation reduction at $7.5 \mathrm{fps}$ remained significant after controlling for the number of angiograms and the case type, further emphasizing the importance of lowering the PFR to minimize radiation exposure. Equally importantly, lower PFR was not associated with any perceived differences in procedural difficulty or length, and there were no complications related to the lower PFR.

Because many patients with congenital heart disease undergo multiple cardiac catheterization procedures, they are exposed to ionizing radiation, which can have both immediate and long-term effects. The negative consequences of ionizing radiation can also be categorized as either deterministic or stochastic effects. While deterministic effects, like cataract formation or skin injury, have a somewhat predictable dose-response relationship, with degree of injury directly correlating with absorbed radiation dose, stochastic effects, like cancer and genetic mutations, are unpredictable without threshold effect. ${ }^{4}$ Strides have been made, particularly in the past decade, in improving radiation safety profiles as well as public awareness. Initiatives such as the ALARA (as low as reasonably achievable) conference, conducted by the Society for Pediatric Radiology in 2006, concluded that fluoroscopy dose optimization and reduction were key areas of concern. ${ }^{5}$ Campaigns such as "Image Gently, Step Lightly," first launched in August of 2009, incorporated a standard safety checklist to encourage proper preparation, technique, and strategies to lower radiation exposure. ${ }^{6}$ In particular, this checklist encouraged utilizing pulse fluoroscopy rather than continuous fluoroscopy when possible, as well as the using the lowest pulse rate possible.

In this study, we did not see a further reduction in radiation exposure in the 5-fps group compared to the 7.5-fps group. However, there were also no differences in the impact of PFR on procedure length or case difficulty between these groups. Due to our study design and intent-to-treat analysis, physicians were able to lower the set PFR during procedures. In fact, some patients (6 of 25 , $24 \%$ ) in the 7.5 -fps group actually received a lower fluoroscopy rate. For this reason, 5 fps may still offer potential radiation-lowering advantages over 7.5 fps. Therefore, continuing to strive for ultralow radiation exposure in pediatric cardiac 
catheterization procedures should be a benchmark for the highest-quality care.

A limitation of our study was the potential for interphysician practice variation (apart from starting PFR) that could impact radiation exposures. The use of lead screens and shields, as well as awareness and incorporation of best practices for minimizing radiation exposure, can vary from physician to physician. In an effort to examine the impact of this practice heterogeneity, we compared providers within the 7.5 -fps group and found no group difference in radiation exposures. While this does not mean that other radiationlowering techniques are not important in lowering radiation exposure, it shows that in this study population, they had less impact on lowering radiation doses compared with lowering the PFR.

While lowering the baseline PFR is a first step toward radiation reduction in pediatric cardiac catheterization procedures, further efforts to lower radiation exposure of both patients and physicians might include lowering the frame rate of cineangiograms, more consistent use of radiation barriers like lead shields, and using nonfluoroscopic imaging techniques as adjunctive tools during catheterization procedures. Well-planned, well-executed quality improvement studies might provide both insight into and progress in truly achieving the lowest achievable radiation dose for patients.

Although not the primary focus of the study, we found that the case-specific dosimeters were an excellent surrogate marker for patient radiation exposure. Thus, the use of similar dosimeters offers a more direct way to measure radiation exposure, and although the devices cannot measure deep tissue exposure, the potential to place these devices on patients and directly measure skin exposure may improve our ability to measure the effect of radiation-lowering efforts in the future.

\section{Conclusions}

This quality improvement study revealed that a starting PFR of 7.5 fps was associated with a significant reduction in radiation exposure compared with $15 \mathrm{fps}$ with no adverse effects. For this reason, we have changed the practice of all attending interventionalists in our pediatric cardiac catheterization laboratory so that the default starting fluoroscopy rate for every procedure is set to $7.5 \mathrm{fps}$.

\section{Author Contributions}

Stuart H. Covi was involved in the concept and design, data collection/interpretation, and drafting the article. Wendy Whiteside was involved in data collection and in critical revision and approval of the article. Sunkyung Yu was involved in the concept and design, the data analysis/ interpretation, and the critical revision and approval of the article. Jeffrey D. Zampi was involved in the concept and design, the data collection/interpretation, and the critical revision and approval of the article.

\section{Acknowledgments}

We thank Karl Fischer and the University of Michigan radiation safety committee for their efforts in obtaining dosimetry badges and their academic contributions.

Corresponding Author: Jeffrey D. Zampi, MD, Congenital Heart Center, C.S. Mott Children's Hospital, University of Michigan, Floor 11, Cardiology, 1540 E. Medical Center Drive, Ann Arbor, MI 48109-4204, USA. Tel: +734-936-3813; Fax: +734-936-9470; E-mail: jzampi@med.umich.edu

Conflict of interest: None.

Accepted in final form: May 12, 2014.

\section{References}

1 Brenner DJ, Elliston CD, Hall EJ. Estimated risks of radiation-induced fatal cancer from pediatric CT. Am 7 Radiol. 2001;176:289-296.

2 Schueler B, Julsrud P, Gray J, Stears J, Wu K. Radiation exposure and efficacy of exposure-reduction techniques during cardiac catheterization in children. Am $\mathcal{F}$ Radiol. 1994;162:173-177.

3 Fetterly KA, Mathew V, Lennon R, Bell MR, Holmes DR, Rihal CS. Radiation dose reduction in the invasive cardiovascular laboratory: implementing a culture of philosophy of radiation safety. $7 A C C$ Cardiovasc Interv. 2012;5:866-873.

4 Stewart FA, Hauer-Jensen M, Kleiman NJ, Akleyev AV, Hendry JH, MacVittie TJ. Early and late effects of radiation in normal tissues and organs: threshold doses for tissue reactions and other non-cancer effects of radiation in a radiation protection context. Ottawa: International Commission on Radiological Protection; 2011. ICRP ref 4844-6029-7736.

5 Willis CE. Proceedings of the Second ALARA Conference. Pediatr Radiol. 2004;34:159-248.

6 Sidhu MK, Goske MJ, Coley BJ, et al. Image gently, step lightly: increasing radiation dose awareness in pediatric interventions through an international social marketing campaign. $f$ Vasc Interv Radiol. 2009;20:1115-9. 